\title{
Improving three-dimensional (3D) range gated reconstruction through time-of-flight (TOF) imaging analysis
}

\section{S. Y. Chua}

\section{Wang \\ wang.xin@monash.edu}

N. Guo

C. S. Tan

T. Y. Chai

G. L. Seet

School of Engineering, Monash University Malaysia, Jalan Lagoon Selatan, 47500 Bandar Sunway, Selangor, Malaysia

School of Engineering, Monash University Malaysia, Jalan Lagoon Selatan, 47500 Bandar Sunway, Selangor, Malaysia

School of Engineering, Monash University Malaysia, Jalan Lagoon Selatan, 47500 Bandar Sunway, Selangor, Malaysia

Faculty of Engineering, Multimedia University, Jalan Multimedia, 63000 Cyberjaya, Selangor, Malaysia

Department of Mechatronics and BioMedical Engineering, Universiti Tunku Abdul Rahman, 53300 Setapak, Kuala Lumpur, Malaysia

School of Mechanical and Aerospace Engineering, Nanyang Technological University, Singapore 639798

This paper performs an experimental investigation on the TOF imaging profile which strongly influences the quality of reconstruction to accomplish accurate range sensing. From our analysis, the reflected intensity profile recorded appears to deviate from Gaussian model which is commonly assumed and can be perceived as a mixture of noises and actual reflected signal. Noise-weighted Average range calculation is therefore proposed to alleviate noise influence based on the signal detection threshold and system noises. From our experimental result, this alternative range solution demonstrates better accuracy as compared to the conventional weighted average method and proven as a para-axial correction to improve range reconstruction in 3D gated imaging system.

[DOI: http://dx.doi.org/10.2971/jeos.2016.16015]

Keywords: Range sensing, 3D imaging, noise influence

\section{INTRODUCTION}

Laser ranging technology has developed rapidly to become one of the crucial sensorial assets in industrial and day-to-day applications. This has been a great interest in optical metrology because of its non-contact and non-destructive nature [1]. Due to the high speed and long range capability offered by laser, it has been deployed in miscellaneous fields such as remote sensing, machine vision, 3D imaging, etc [2]-[4].

Range gated is a potential range sensing approach based on TOF principle, by measuring the round trip time between laser pulse emission and the return of the pulse echo resulting from its reflectance off the target. Range $r$ can be determined from the two-way travel time $t$ and the speed of light $c$.

$$
r=\frac{c t}{2}
$$

This approach controls the pulsed laser in conjunction with the camera's shutter speed to capture the reflectivity images from the target scene. It has been a promising imaging method used in many applications such as target detection and recovery [5], long range imaging [6], night vision [7, 8], underwater $[9,10]$ and 3D imaging $[11,12]$. Moreover, range gated approach has become cheaper and affordable due to the continuous development in lasers, sensors, signal processing, and computer technology. Besides the system enhancement, accu- racy improvement in term of range computation is also critical especially in some cases where the performance is limited by hardware capability.

Reflected laser pulse returns to the incoming direction after strikes on a target, which contains the key information for range determination in TOF $3 \mathrm{D}$ reconstruction. Since the quality of reconstruction highly relies on the TOF imaging profile, it is necessary to study the characteristics of reflected laser pulse. The importance of intensity profile has been emphasised and studied in various applications [13, 14]. System parameters can be analysed from the laser profile, for instance, the laser and receiver models, atmospheric effects, which are helpful for result interpretation and prediction. In this context, energy profile in term of variation and redistribution of laser pulse energy needs to be well understood to perform accurate range computation. Gaussian model has been the most common assumption used due to its simplicity and efficacy [15]-[17]. Alda suggested that Gaussian is a reference of quality for a laser source which needs to be extended to define its distribution [18]. In general, the returned waveform can be modelled as a sum of Gaussian pulses [19]. Therefore, the signal can be assumed as a mixture of Gaussian distributions [20], Lognormal, and generalised Gaussian model [21]. How- 
ever, no attempt was made to further explore the TOF imaging profile in a range gated system.

In practice, the reflected intensity recorded deviates from the actual target echo due to various sources of error and noise [22]. The performance of Intensified Charged Coupled Device (ICCD) camera as the detector in the range gated imaging system can be limited by signal inherent shot noise (i.e. input or photon noise), dark current, and readout noise. In addition, speckle and scintillation noise are the extrinsic noises due to the laser-target interaction and laser beam propagation through the atmosphere [23]. The diffuse light generates a background power at the camera which corresponds to a background pixel value [24]. Images averaging is often applied to reduce the measurement uncertainty caused by random noises where the averaging of $n$ images gives accuracy improvement by $\sqrt{n}$ factor [25]. However, this does not improve in term of the systematic error in a range gated system which can be due to imperfections of illumination, intensity errors caused by low or over-exposed condition, etc [22].

In this paper, we investigate the reflection profile via sequence of two-dimensional (2D) gated images. An experimental study on the 3D range gated reconstruction is carried out to propose solution at para-axial condition. Alternative range computation is suggested based on the TOF imaging profile and noise analysis with accuracy comparison to the conventional weighted average method.

\section{RANGE GATED IMAGING SYSTEM SETUP}

The schematic diagram of the range gated imaging system for 3D reconstruction is shown in Figure 1(a). Figure 1(b) shows the experimental setup of our range gated imaging system. The major components include a pulsed laser system, ICCD camera and its control unit, delay generator, power supplies, lens assemblies, and frame grabber. A Q-switched Nd:YAG laser with wavelength $532 \mathrm{~nm}$ and pulse width $\sim 10 \mathrm{~ns}$ provides illumination to the target. ICCD gated camera works as the receiver where the control of the camera gate opening and gate duration is important, and frame grabber captures the reflectivity images from the camera for $3 \mathrm{D}$ reconstruction. The camera gate opening is triggered by delay generator and the gate duration can be adjusted from $5 \mathrm{~ns}$ to $20 \mathrm{~ms}$. Synchronisation between the laser emission and camera capture is controlled through the delay generator setup where the camera gate signal triggers the high speed shutter to open for $\sim 10 \mathrm{~ns}$ and is time delayed with a time step of 100 ps to capture a sequence of $2 \mathrm{D}$ gated images. In this context, the two-way travel time can be determined to obtain the range or depth for each pixel of the object based on TOF equation. Accordingly, the object surface can be reconstructed in 3D form from the calculated depth map.

\section{TIME-OF-FLIGHT IMAGING ANALYSIS}

In a range gated imaging system, the total energy received and captured in an image pixel is the incident energy of laser pulse

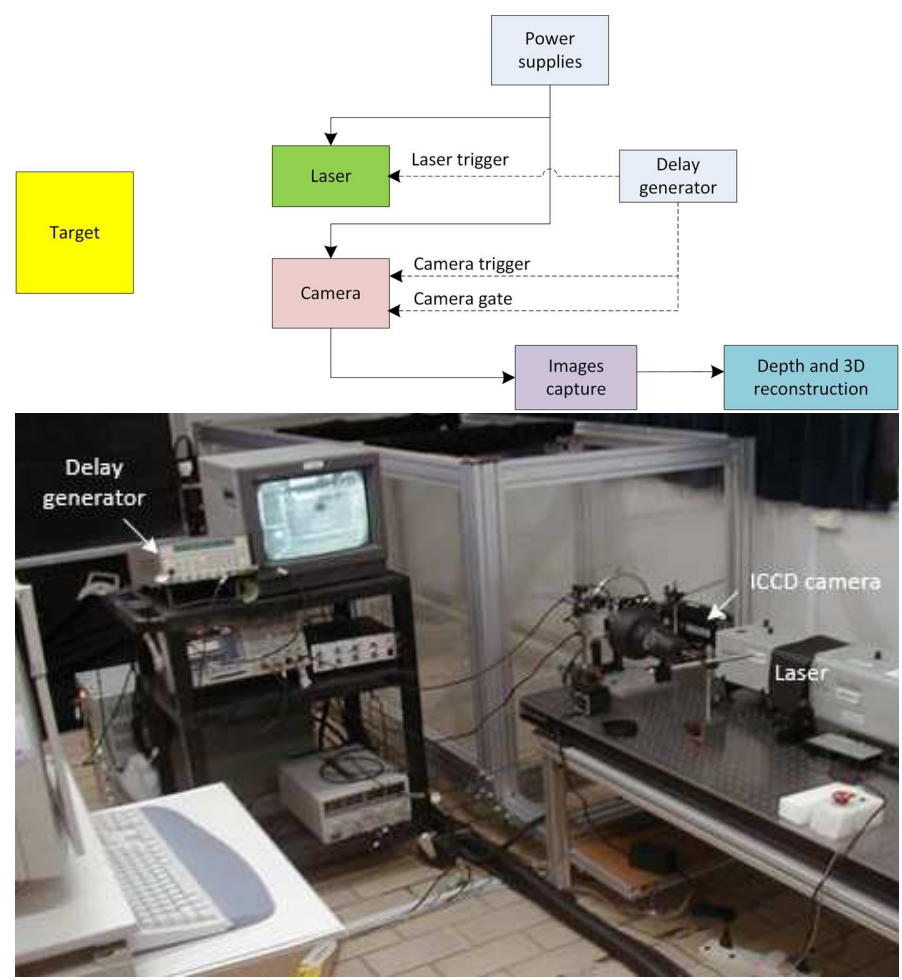

FIG. 1 Range gated imaging system setup. (a) Schematic diagram of range gated imaging system for $3 \mathrm{D}$ reconstruction. (b) Experimental setup of range gated imaging system.

integrated during the camera gate opening. The reflected laser pulse $P(t)$ is delayed by the round trip travel time $\frac{2 r}{c}$ and the camera gate $G(t)$ is delayed by time $t_{i}$ sequentially with a time step $t_{\text {step }}$ based on time slicing technique [26]. $i$ denotes the number of image slices captured in sequence where $i=1,2, \ldots, n$.

$$
I_{i}=\int P\left(t-\frac{2 r}{c}\right) G\left(t-t_{i}\right) d t
$$

Camera gate function is often assumed as $G(t)=1$ when $0 \leq t \leq t_{\text {gate }}$, where $t_{\text {gate }}$ is the camera gate time.

Range gated imaging is treated as one pixel problem because the reflected energy from the target scene is a function of various influencing factors such as illuminating laser profile, bidirectional reflection distribution function (BRDF), which vary across the target scene [6]. Theoretically, each pixel should exhibit the same characteristic with the reflected laser pulse. The temporal profile of the reflected laser pulse $P(t)$ is commonly approximated as Gaussian form [26].

$$
P(t)=\frac{P_{o}}{\sqrt{2 \pi} \sigma_{p}} \exp \left(\frac{-t^{2}}{2 \sigma_{p}^{2}}\right)
$$

$P_{o}$ is the maximum transmitted energy and $\sigma_{p}$ is the standard deviation of the echo pulse. To investigate the energy distribution, a sequence of time delayed images can be analysed for individual pixels. Figure 2(a) and 2(c) show the temporal profile of two example pixels while Figure 2(b) and 2(d) show the probability distribution fitting result of the example pixels. The blue circles represent the data of pixel intensity and the red line indicates the ideal Gaussian fit. It can be seen that distribution of Pixel1 is approximately Gaussian whereas distribution of Pixel2 shows significant deviation and is not well 
a)
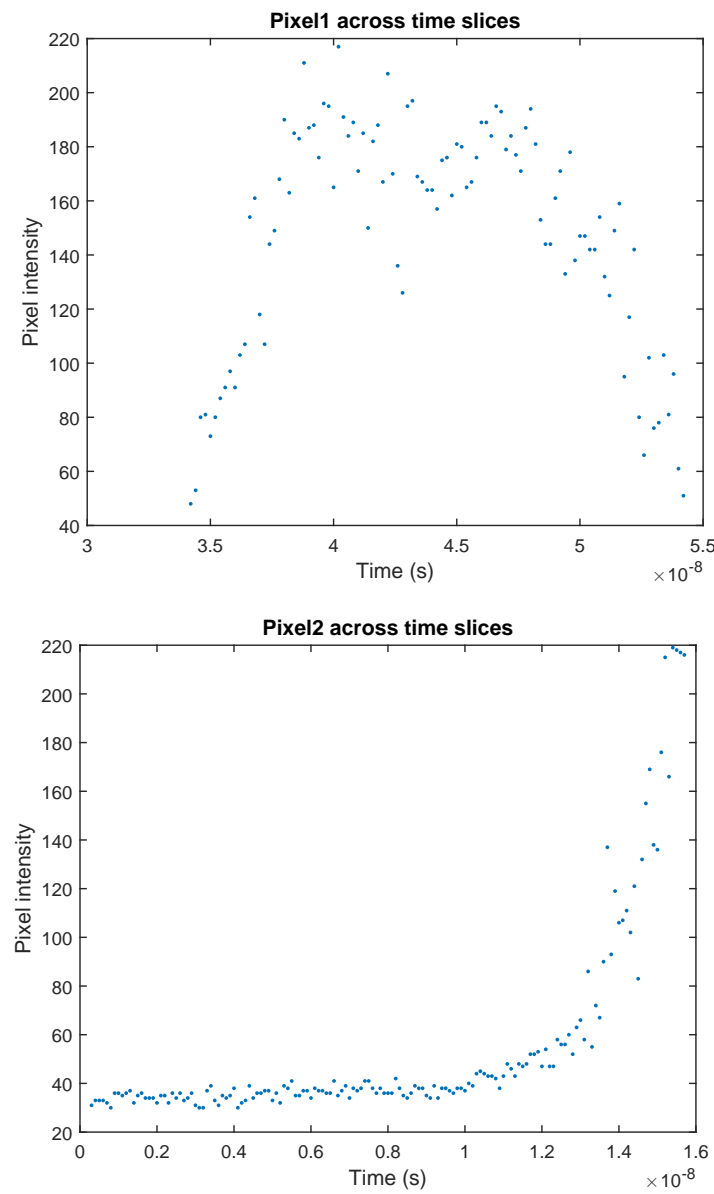

b)
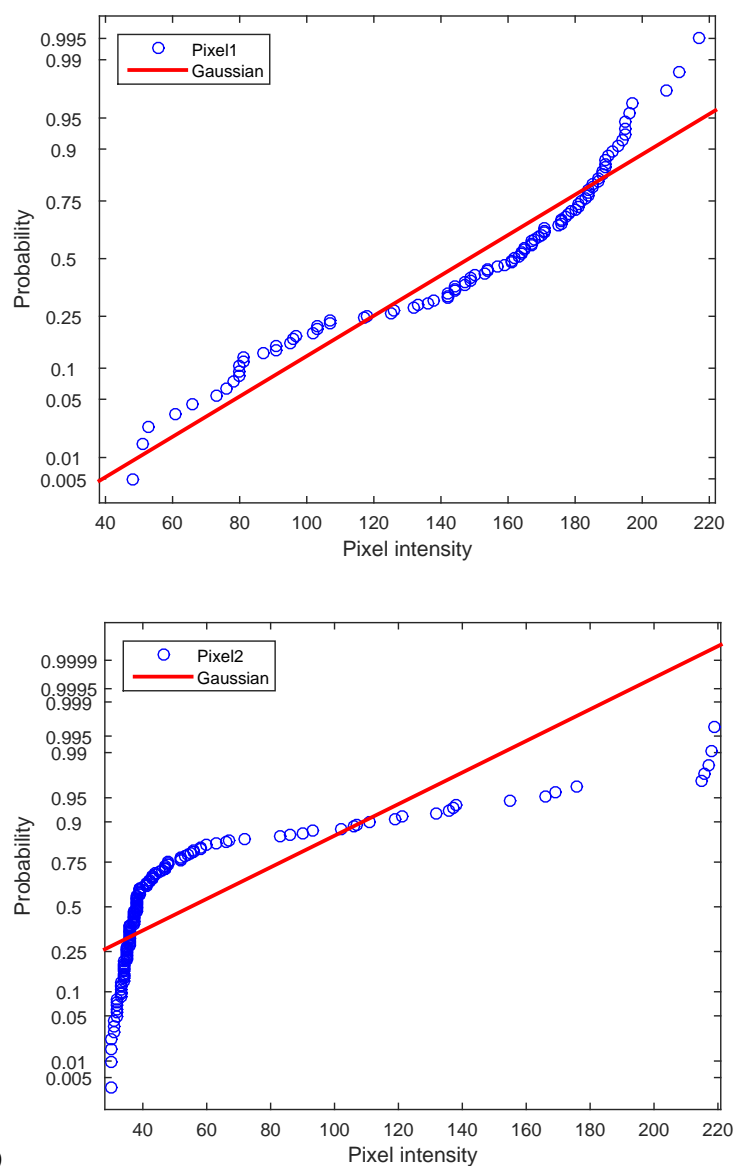

FIG. 2 Temporal profile and probability fitting for two example pixels reflected from the targeted object. Figure 2(a) and 2(b) show an object pixel which behaves approximately Gaussian distributed while Figure 2 (c) and 2(d) correspond to an object pixel exhibits non-Gaussian characteristic. (a) Temporal profile for Pixel1. (b) Probability fitting for Pixel1. (c) Temporal profile for Pixel2. (d) Probability fitting for Pixel2.

fitted to Gaussian model as compared to Pixel1. This shows that the distribution of pixel intensity can deviate from pure Gaussian model.

The TOF imaging profile is disturbed by multiple sources of noises in practice. The pixel intensity captured thus is a noisy sample from the convolution function in Eq. (2). Pixel value or the total energy on each image pixel $I_{i}$ can be expressed as the sum of different sources including the contributions from target reflection $I_{\text {target }}$, atmospheric backscatter $I_{b s c}$, background $I_{\text {backgr }}$ and detector noise $I_{\text {noise }}$ [24].

$$
I_{i}=I_{\text {target }}+I_{b s c}+I_{\text {backgr }}+I_{\text {noise }}
$$

Signal-to-noise ratio (SNR) is defined as the ratio between detected intensity $I_{i}$ and associated noises $\delta I_{i}$.

$$
S N R=\frac{\sum_{i} I_{i}}{\sqrt{\sum_{i}\left(\delta I_{i}\right)^{2}}}
$$

In general, SNR is inversely proportional to $\delta I_{i}$ contributed by $I_{b s c}, I_{\text {backgr }}$, and $I_{\text {noise }}$. Therefore, SNR can be controlled and improved by suppressing the amount of associated noises.

We can estimate the background $I_{\text {backgr }}$ and detector noise $I_{\text {noise }}$ by capturing an image without the presence of target reflection. An example of distribution fitting for Pixel2 after exclusion of noise data can be seen in Figure 3 which shows the distribution of the actual reflected signal of image pixel is

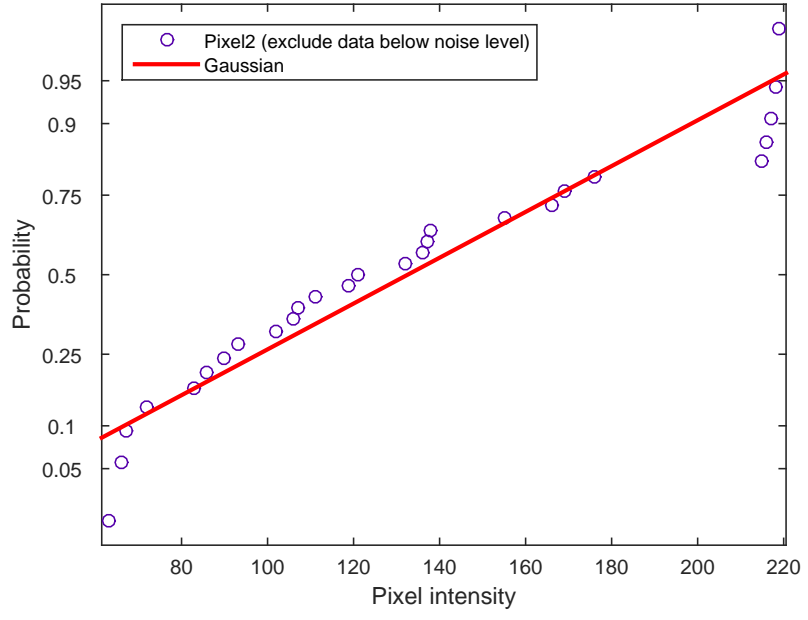

FIG. 3 Probability fitting for Pixel2 after noise data elimination.

closer to Gaussian model without the noise influence. However, it can be seen that there are still outliers exist mainly in the low and high intensity regions. System noise partly contributed by the detector noise and background can be the reason for outliers in the low intensity region while the outliers in the high intensity region possibly caused by the over-exposed pixel or spike noise, which are considered in the proposed range computation. 


\section{PROPOSED RANGE CALCULATION MODEL}

When time slicing technique is used for image acquisition and reconstruction, the step size $t_{\text {step }}$ for image sequence captured is much smaller compared to the laser pulse width and camera gate i.e. $t_{\text {ste }} \ll \sigma_{p}, t_{\text {ste }} \ll t_{\text {gate }}$. The summation of radiant energy in an image pixel $I(x, y)=\sum_{i} I_{i}(x, y)$ where the summation $\sum_{i}$ can be seen as the integration over time slices $\int d t_{i} / t_{\text {step }}[26]$.

$$
I=\sum_{i} I_{i}=\frac{\int I_{i} d t_{i}}{t_{\text {step }}}
$$

Conventional weighted average method has been an effective yet simple implementation for range calculation. The average two-way travel time $\langle t>$ to a field-of-view point or pixel can be obtained as [26, 27]:

$$
<t>=\frac{\sum_{i=1}^{n} I_{i} t_{i}}{\sum_{i=1}^{n} I_{i}}
$$

where $\sum_{i=1}^{n} I_{i}$ is the summation of $n$ reflected laser pulse intensity or the $(\mathrm{x}, \mathrm{y})$ pixel value for $n$ gated images, and $I_{i}$ is the intensity or the $(\mathrm{x}, \mathrm{y})$ pixel value at delayed time $t_{i}$ (or equivalent $i$ th gated image). Accordingly, the depth or range of the fieldof-view point or pixel can be reconstructed from $r=\frac{c<t>}{2}$ based on TOF principle. Weighted average method is a suitable approach for Gaussian distributed data, however, TOF imaging profile as investigated is not a true Gaussian under noise influence. Therefore, it is possible to improve range accuracy using alternative computation model.

As discussed in Section 3, noise is unavoidable causing the received intensity to deviate from ideal Gaussian distribution. Typically, noise observed at low intensity and spike artefact or over-exposure occurs at high intensity region are the outliers. Rather than remembering all of these noisy details, it makes sense to down-weight the influence or lower the probability of false alarm in the intensity ranges where noise is dominant. Nevertheless, we are more interested in the higher intensity data returned that likely determine the detected range. We propose a method which we refer as Noise-weighted Average to refine the range calculation:

$$
<t>=\frac{\sum_{i=1}^{n} w_{i} I_{i} t_{i}}{\sum_{i=1}^{n} w_{i} I_{i}}
$$

where $w_{i}, 0 \leq w_{i} \leq 1$ is defined as noise-weighted coefficient. When $w_{i}=1$ for $i=1,2, \ldots, n$, this model is the same as the conventional weighted average method assuming noise is negligible. $I_{i}$ represents the intensity or the $(\mathrm{x}, \mathrm{y})$ pixel value at delayed time $t_{i}$ (or equivalent $i$ th gated image), and $\sum_{i=1}^{n} I_{i}$ is the summation of intensity or pixel value for $n$ gated images.

The evidence presented thus far demonstrates the need for better strategy to handle the reflected laser pulse data. We make a simplified assumption of normally distributed noise that is independent of the reflected signal and is excluded

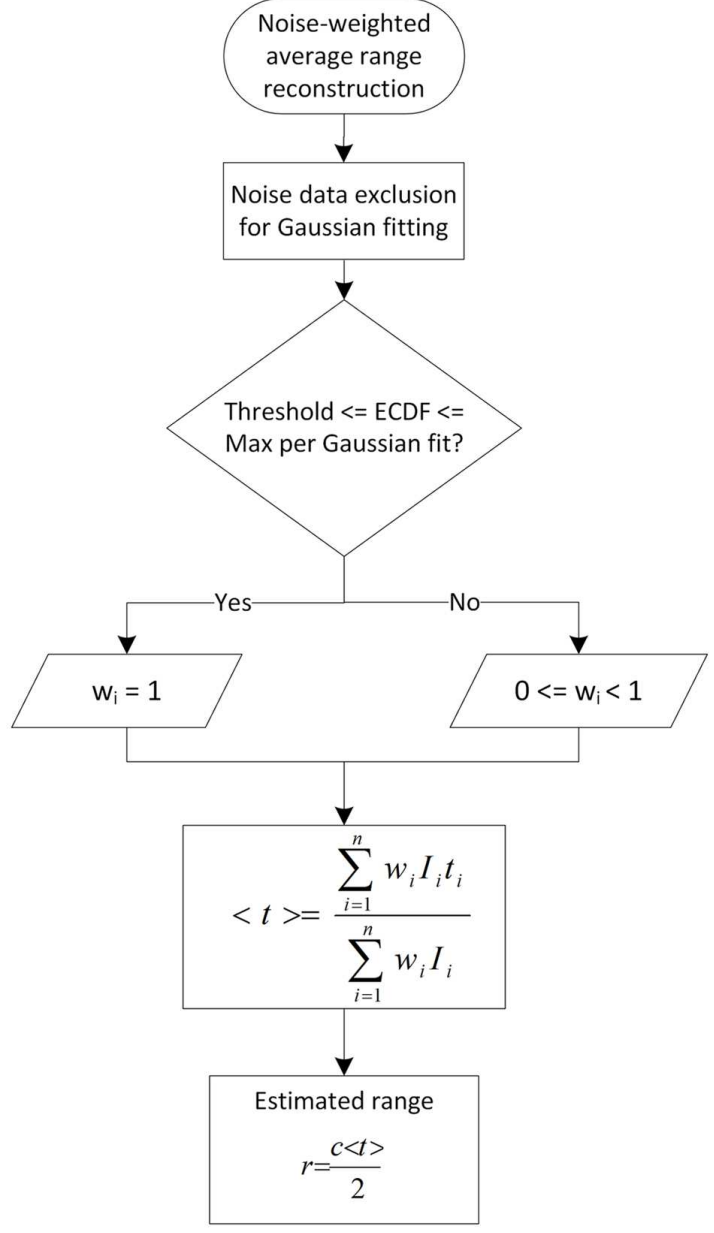

FIG. 4 Flow chart of range gated reconstruction algorithm.

so that the returned data fits better to Gaussian distribution. The outliers can be identified based on the re-fitted Gaussian model. The computation scheme is illustrated in Figure 4 . Firstly, the captured intensity in the form of image pixel data is processed to exclude system noise identified. The resulted data is fitted to Gaussian distribution model and maximum Empirical Cumulative Distribution Function (ECDF) value is obtained. Essentially, when $w_{i}=1$, noise is assumed to be negligible with higher probability for detection meanwhile $0 \leq w_{i}<1$ is applied to data with higher noise probability to reduce the influence of noises.

$$
w_{i}= \begin{cases}w_{i}=1 & \text { if Threshold } \leq \mathrm{ECDF} \\ 0 \leq w_{i}<1 & \leq \text { Max per Gaussian fit } \\ & \text { otherwise, noise influence is } \\ & \text { considered }\end{cases}
$$

Intensity threshold is to control the probability of detection and noise-weighted coefficient is applied to indicate the reliability of measurement, which both can be chosen independently based on the prior knowledge of the detection limit and system noise. In this context, the threshold set must be above the noise level i.e. $>I_{b s c}+I_{b a c k g r}+I_{\text {noise }}$. The proposed computation model is verified in the following section. 


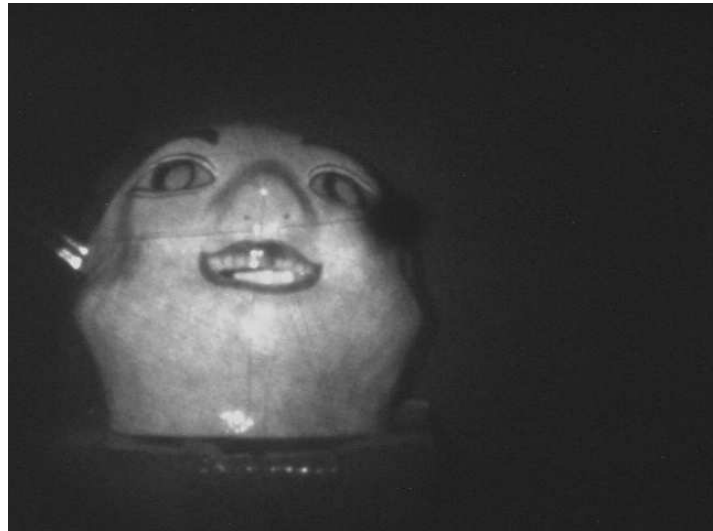

FIG. 5 Raw image of 0bject 1 captured by the range gated imaging system.

\section{3D RANGE GATED SURFACE RECONSTRUCTION}

Our experiment is conducted in a controlled environment where the illumination and atmospheric effects are negligible. Figure 5 shows the raw gray-scale image captured by the range gated imaging system. A sequence of $2 \mathrm{D}$ gray-scale images is captured by the ICCD camera across the target scene. The range value for each pixel is determined separately since range gated imaging is treated as one pixel problem and eventually constructs the 3D surface of the target scene. Test object with depth measurement $0.48 \mathrm{~m}$ from background is reconstructed from a total of 155 gated images with time step 100 ps. As discussed in Section 3, the TOF imaging profile of image pixels could be non-Gaussian distributed because of the noise data. To reduce the influence of noise anticipated at lower intensity region, one can adapt the aforementioned Noise-weighted Average model to down-weight the noisy images. Smaller noise-weighted coefficient should be applied when the noise probability is higher.

From Eq. (4), the total noise in the system is given by $I_{b s c}+$ $I_{\text {backgr }}+I_{\text {noise }}$. Therefore, we need to alleviate the noise influence at low intensity region. For our verification, threshold is selected above the total noise level as half of the maximum intensity based on full width at half maximum (FWHM) criterion of laser pulse and noise-weighted coefficient of 0.5 is used in Eq. (8) for range calculation. Result of the object depth reconstruction in Table 1 shows that the Noise-weighted Average method yields distinctly smaller depth error of $3.84 \%$ as compared to the weighted average method which gives a depth error of $12.65 \%$. Graphical representation of the 3D surface reconstruction is shown in Figure 6. Suffice to say, it is possible to produce better reconstruction accuracy with the para-axial correction on reflected laser pulse profile using Noise-weighted Average method as an alternative to the conventional weighted average method.

\begin{tabular}{cc}
\hline $\begin{array}{c}\text { Weighted } \\
\text { Average }\end{array}$ & $\begin{array}{c}\text { Noise-weighted } \\
\text { Average }\end{array}$ \\
\hline $12.65 \%$ & $3.84 \%$
\end{tabular}

TABLE 1 Absolute depth error (\%) comparison between weighted average and Noiseweighted Average methods.
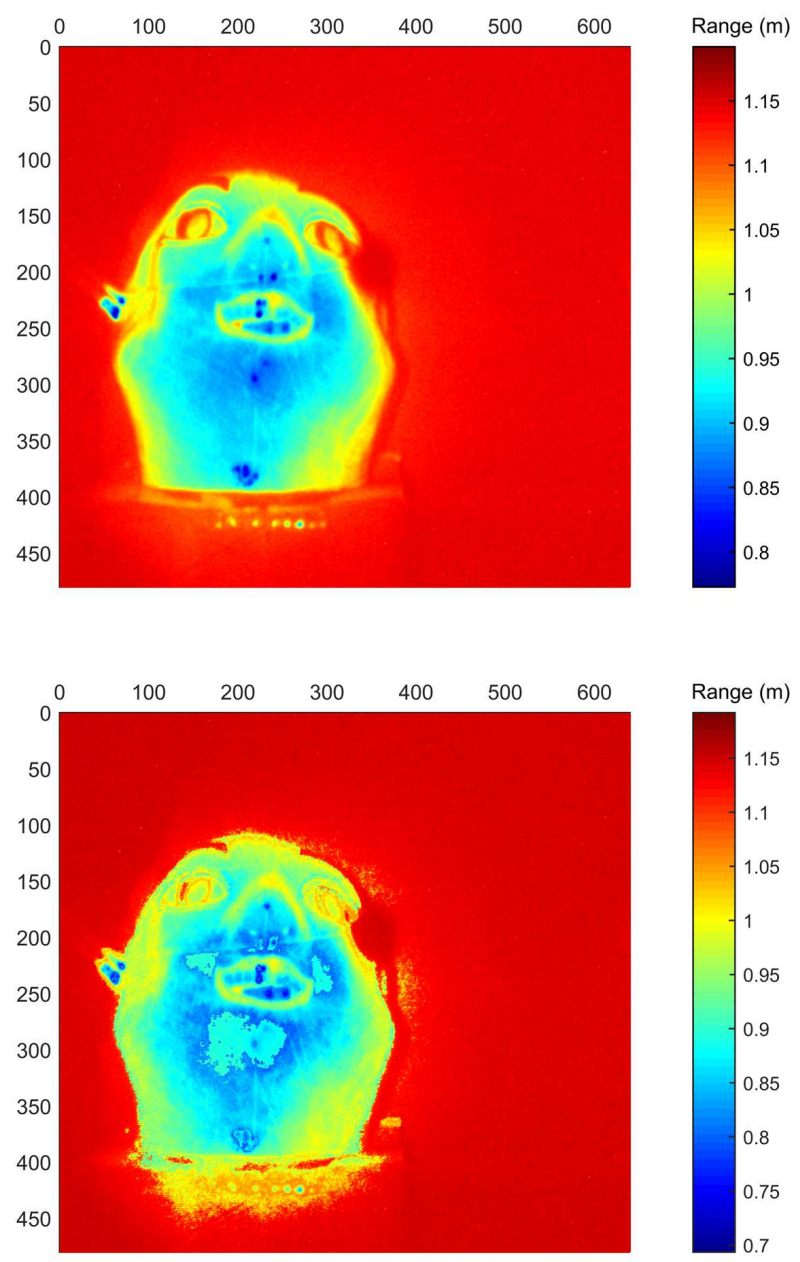

FIC. 6 Comparison of $3 \mathrm{D}$ surface reconstruction using conventional weighted average and Noise-weighted Average range calculation models. (a) Reconstructed object1 based on conventional weighted average method. (b) Reconstructed object1 based on Noise-weighted Average method.

\section{CONCLUSION}

In this paper, we reveal that the TOF imaging profile in $3 \mathrm{D}$ range gated reconstruction exhibits non-Gaussian characteristics under noise influences. This finding is important as the TOF range gated technique relies on the reflectivity images for $3 \mathrm{D}$ reconstruction. The reflected intensity profile can be perceived as a mixture of noises and actual signals. We propose the Noise-weighted Average method to refine the range calculation based on intensity threshold to control the probability of detection and noise-weighted coefficient to indicate the reliability of intensity measurement. As evident from the experimental result, the proposed method to alleviate the noise influence yields better range estimation as compared to the conventional weighted average method. This correction is valid for the reflected laser pulse profile that arrives close to the para-axial condition. Para-axial condition limits the images capture to be very close to the lens axial but some constraints may enlarge the deviation or change the reflected laser pulse profile eventually.

In future, optimisation on the Noise-weighted Average method can be further looked into to improve its efficiency 
and robustness by modelling the reflected signal with more complex distribution models such as Lognormal and generalised Gaussian. Additionally, other effects caused by target reflectivity and detector are to be investigated and compensated in the range reconstruction algorithm.

\section{ACKNOWLEDGEMENTS}

The authors gratefully acknowledge the support and funding from Monash University Malaysia and Ministry of Higher Education, Malaysia under the Grant No: FRGS/1/2013/SG02/MUSM/02/1.

\section{References}

[1] P. Huke, R. Klattenhoff, C. von Kopylow, and R. Bergmann, “Novel trends in optical non-destructive testing methods," J. Eur. Opt. Soc.-Rapid 8, (2013).

[2] M. Amann, T. Bosch, M. Lescure, R. Myllyla, and M. Rioux, "Laser ranging: a critical review of usual techniques for distance measurement," Opt. Eng. 40, 10-19 (2001).

[3] E. Pike, "Lasers, photon statistics, photon-correlation spectroscopy and subsequent applications," J. Eur. Opt. Soc.-Rapid 5, (2010).

[4] X. Su and Q. Zhang, "Dynamic 3-D shape measurement method: a review," Opt. Laser. Eng. 48, 191-204 (2010).

[5] A. Velten, T. Willwacher, 0. Gupta, A. Veeraraghavan, M. G. Bawendi, and R. Raskar, "Recovering three-dimensional shape around a corner using ultrafast time-of-flight imaging," Nature communications 3, 745 (2012).

[6] P. Andersson, "Long-range three-dimensional imaging using range-gated laser radar images," Opt. Eng. 45, 034301 (2006).

[7] D. Monnin, A. L. Schneider, F. Christnacher, and Y. Lutz, "A 3D Outdoor Scene Scanner Based on a Night-Vision Range-Gated Active Imaging System," in Proceedings to Third International Symposium on 3D Data Processing, Visualization, and Transmission (3DPVT'06), 938-945 (IEEE, Chapel Hill, 2006).

[8] X.-W. Wang, Y. Zhou, S.-T. Fan, J. He, and Y.-L. Liu, “Range-Gated Laser Stroboscopic Imaging for Night Remote Surveillance," Chinese Phys. Lett. 27, 094203 (2010).

[9] J. Busck, "Underwater 3-D optical imaging with a gated viewing laser radar," Opt. Eng. 44, 116001 (2005).

[10] F. Caimi and F. Dalgleish, "Performance considerations for continuous-wave and pulsed laser line scan (LLS) imaging systems," J. Eur. Opt. Soc.-Rapid 5, (2010).

[11] X. Zhang and H. Yan, "Three-dimensional active imaging with maximum depth range," Appl. Optics 50, 1682-1686 (2011).
[12] X. Wang, Y. Li, and Y. Zhou, "Triangular-range-intensity profile spatial-correlation method for 3D super-resolution range-gated imaging," Appl. Optics 52, 7399-7406 (2013).

[13] R. Olsson, I. Eriksson, J. Powell, and A. Kaplan, "Advances in pulsed laser weld monitoring by the statistical analysis of reflected light," Opt. Laser. Eng. 49, 1352-1359 (2011).

[14] Y. Zhao, A. MR, and X. Wang, "Influence Factor Analysis for 3D Imaging Laser Radar Range Profile," Middle-East Journal of Scientific Research 17, 142-147 (2013).

[15] S. Kruapech and J. Widjaja, "Laser range finder using Gaussian beam range equation," Opt. Laser Technol. 42, 749-754 (2010).

[16] R. Filter, T. Scharf, and H. P. Herzig, “High resolution displacement detection with speckles: accuracy limits in linear displacement speckle metrology," J. Eur. Opt. Soc.-Rapid 5, (2010).

[17] J. Wang, S. Zhao, G. Li, K. Yang, D. Li, J. An, and M. Li, “Analysis of output characteristics of the actively Q-switched intracavity optical parametric oscillator considering Gaussian distribution of intracavity photon densities," J. Mod. Opt. 55, 1267-1277 (2008).

[18] J. Alda, "Laser and Gaussian beam propagation and transformation," in Encyclopedia of optical engineering R. G. Driggers, C. Hoffman, and R. Driggers, eds., 999-1013 (CRC Press, Boca Raton, 2003).

[19] W. Wagner, A. Ullrich, V. Ducic, T. Melzer, and N. Studnicka, “Gaussian decomposition and calibration of a novel small-footprint fullwaveform digitising airborne laser scanner," ISPRS J. Photogramm. 60, 100-112 (2006).

[20] C. Mallet and F. Bretar, "Full-waveform topographic lidar: State-ofthe-art," ISPRS J. Photogramm. 64, 1-16 (2009).

[21] A. Chauve, C. Mallet, F. Bretar, S. Durrieu, M. P. Deseilligny, and W. Puech, "Processing full-waveform lidar data: modelling raw signals," Int. Soc. Photogramme. 2007, 102-107 (2007).

[22] G. Tsagkatakis, A. Woiselle, G. Tzagkarakis, M. Bousquet, J.-L. Starck, and P. Tsakalides, "Active range imaging via random gating," Proc. SPIE 8542, 85420P-85420P (2012).

[23] R. L. Espinola, B. Teaney, Q. Nguyen, E. L. Jacobs, C. E. Halford, and D. H. Tofsted, "Active imaging system performance model for target acquisition," Proc. SPIE 6543, 65430T-65430T (2007).

[24] O. Steinvall, H. Olsson, G. Bolander, C. A. Groenwall, and D. Letalick, "Gated viewing for target detection and target recognition," Proc. SPIE 3707, 432-448 (1999).

[25] 0. Steinvall, T. Chevalier, P. Andersson, and M. Elmqvist, "Performance modeling and simulation of range-gated imaging systems," Proc. SPIE 6542, 654218-654218 (2007).

[26] J. Busck and H. Heiselberg, "Gated viewing and high-accuracy three-dimensional laser radar," Appl. Optics 43, 4705-4710 (2004).

[27] L. Li, L. Wu, X. Wang, and E. Dang, “Gated viewing laser imaging with compressive sensing," Appl. Optics 51, 2706-2712 (2012). 